\title{
What Fascinates Me in Science ...
}

\author{
Sudeep Gupta ${ }^{1 \odot}$ \\ ${ }^{1}$ Department of Medical Oncology, Advanced Centre for Treatment \\ Research and Education in Cancer (ACTREC), Tata Memorial Centre, \\ Mumbai, Maharashtra, India
}

Ind J Med Paediatr Oncol 2021;42:484-485.
Address for correspondence Sudeep Gupta, MD, DM, Department of Medical Oncology, Advanced Centre for Treatment Research and Education in Cancer (ACTREC), Tata Memorial Centre, Mumbai 410210, Maharashtra, India (e-mail: sudeepgupta04@yahoo.com).
The PhD students of my institution organize an annual conference called the National Research Scholars' Meet. The theme of 2019 meeting was the quintessential dilemma that faces every serious researcher at some point in her career-should she apply existing knowledge for a useful outcome or try to discover new truths about nature? There is no correct answer and sometimes the answer is both! This theme set me thinking about which discoveries in science have fascinated me the most. Here is my list of the most intellectually stimulating scientific feats of the past 200 years. I must state that this "list" is by no means comprehensive nor is it the only one possible. It is mine.

In physical and mathematical sciences, I rank Bernhard Reimann's conceptualization of the mathematics and theory of curvature tensor in differential geometry on the top of my list. This is followed by Heisenberg's foundational work leading to quantum mechanics. Then comes Einstein's General Relativity (which is actually derived from Reimann's work), followed by de Broglie, Schrodinger, Pauli, and Dirac's work on particles and wave functions. Of course, there have been many other great feats in the realm of physical and mathematical sciences, the most recent being Andrew Wiles' proof of Fermat's Last Theorem, ${ }^{1,2}$ until recently the most famous unsolved problem in mathematics.

In the life sciences domain, on top of my list is Gregor Mendel's magical derivation of genetic hereditary principles from study of plants. This, in my opinion, has to be the greatest intellectual feat of all time in life sciences. Then are Charles Darwin and Russel Wallace's enunciation of the theory of evolution, Louis Pasteur and Robert Koch's work in microbiology, and finally Rosalind Franklin, James Watson, Francis Crick, and Maurice Wilkins' work leading to discovery of the DNA structure.
I have read some of Steven Weinberg and especially like his Dreams of a Final Theory. ${ }^{3}$ I like his way of classifying intellectual feats in theoretical physics. He says that some discoveries are "sage like." They are important and wonderful insights that change the course of human thought, but it is likely that other people working in the same manner as the discoverer would have made the same discovery. Moreover, the discoverer can explain to others the logical chain of thought that led to the discovery. He classifies Einstein's general relativity as "sage like." Interestingly, Einstein superimposed physical concepts on Reimann's abstract mathematics of curved spaces, which was formulated several decades earlier-of course, this also required an extremely creative mind like Einstein's! Reimann is not the only example of mathematicians' uncanny ability to create beautiful abstract constructs that are only much later found to correspond to the physical reality of nature, but is possibly the most fateful. In the case of Fermat's Last Theorem (no three positive integers $\mathrm{x}, \mathrm{y}$, and $\mathrm{z}$ can satisfy the equation $\mathrm{x} n+\mathrm{y} n=$ $\mathrm{z} n$, for any value of $\mathrm{n}$ greater than 2 ), which had defied proof for more than 350 years, Wiles worked in a "sage like" manner to build on the ideas of several great mathematicians including Yutaka Taniyama, Goro Shimura, André Weil, Gerhard Frey, Jean-Pierre Sierre and Kenneth Ribet, to provide the proof.

The second class of discoveries according to Weinberg is "magical." Even the discoverer finds it difficult to explain how he arrived at the discovery because a logical chain of thought is hard to discern. The brain of the discoverer works in steps (and skips many steps) that are hard to comprehend. Later the discovery can be explained by more conventional wisdom but the process of discovery is like pulling a rabbit out of a hat. He says that Heisenberg's discovery and his
DOI https://doi.org/ $10.1055 / \mathrm{s}-0041-1732824$. ISSN 0971-5851.
(C) 2021. Indian Society of Medical and Paediatric Oncology. All rights reserved.

This is an open access article published by Thieme under the terms of the Creative Commons Attribution-NonDerivative-NonCommercial-License, permitting copying and reproduction so long as the original work is given appropriate credit. Contents may not be used for commercial purposes, or adapted, remixed, transformed or built upon. (https://creativecommons.org/ licenses/by-nc-nd/4.0/)

Thieme Medical and Scientific Publishers Pvt. Ltd., A-12, 2nd Floor, Sector 2, Noida-201301 UP, India 
mathematical steps leading to the formalism of quantum theory (Quantum theoretical re-interpretation of kinematic and mechanical relations, 1925) ${ }^{4}$ were pure magic. In May 1925, Heisenberg suffered from what is possibly the most momentous bout of hay fever in history. To seek relief from his symptoms, he retreated from the flowers and pollen of Gottingen to lonely Helgoland in the North Sea. And there, he rewrote the laws of physics. When he returned, he had the first complete mathematical formulation of the quantum theory. Subsequently, others (most prominently Max Born and Pascual Jordan), working backward, provided more conventional (matrix mechanics) mathematical constructs of quantum mechanics. When I read it in school, I was, and remain until today, spellbound with Heisenberg's 1925 "table of rates."

In the life sciences domain, Mendel's pea experiments were absolutely magical. I have often wondered about his motivations for conducting those meticulous experimentsperhaps the exploits of breeders and farmers inspired him. I think that he had already worked out the laws of segregation and independent assortment in his brain before starting the plant investigations and only undertook the latter to prove his theory. No one in their right mind, in that era, would have conducted the experiments that he painstakingly nurtured over so many years. Almost 150 years later, today, the current genetic paradigm is essentially an extension of Mendel's magic and his genius.

Conflict of Interest

None.

\section{References}

1 Andrew Wiles. Modular elliptic curves and Fermat's Last Theorem. Ann Math 1995;141:443-551

2 Andrew Wiles and Richard Taylor. Ring-theoretic properties of certain Hecke algebras. Ann Math 1995;141:553-572

3 Dreams of a Final Theory. Pantheon, New York: Steven Weinberg; 1992:334

4 Heisenberg W. Uber quantentheoretische Umdeutung kinematischer und" mechanischer Beziehungen. Z Phys 1925; 33:879-893 